\title{
Immune checkpoint inhibitors for hepatocellular carcinoma
}

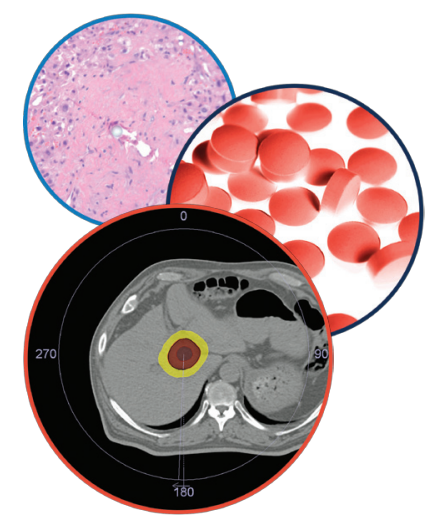

Hepatic Oncology

\section{Practice points}

- Systemic treatment options for advanced hepatocellular carcinoma (HCC) are currently limited to sorafenib, a kinase inhibitor approved in 2007, after demonstrating an increase in overall survival from 7.9 to 10.7 months in a Phase III clinical trial. Several other systemic agents have been trialed in recent years, and none have demonstrated efficacy over sorafenib monotherapy.

- Immune checkpoint inhibitors (monoclonal antibodies targeting coinhibitory molecules on the surface of T cells and antigen presenting cells) have demonstrated evidence of anti-tumor activity in other solid malignancies, particularly melanoma. The most extensively studied of these inhibitors target the CTLA-4 and PD-1 pathways.

- These immune checkpoint pathways play key roles in the physiologic state of relative immune tolerance in the liver, as well as the increased immunosuppression associated with chronic inflammation and HCC. Moreover, increased signaling by these immunosuppressive pathways has been linked to worse outcomes for patients with HCC.

- Emerging clinical data from Phase I/II trials provide preliminary evidence for the effectiveness of these agents for advanced HCC, including in cases of sorafenib treatment failure.

- Further studies of immune checkpoint inhibitors, including a Phase III trial of nivolumab (a PD-1 inhibitor) versus sorafenib in the first-line setting, are either ongoing or slated to begin enrolling patients in the near future.

- Other directions for future research include the combination of immune checkpoint blockade with sorafenib, as well as the identification of predictors of response to checkpoint inhibitors.

Hepatocellular carcinoma (HCC) is a leading cause of cancer deaths worldwide, and advanced HCC generally caries a poor prognosis. The treatment of advanced disease is limited to sorafenib, which provides only a limited improvement in survival, and novel therapies are, thus, sorely needed. Among emerging alternative approaches, immune checkpoint inhibitors are a particularly promising treatment modality. In this review, we summarize current knowledge of the mechanisms for the two primary targets of immune checkpoint inhibitors and discuss the relevance of these pathways to the immunology of HCC. We also review the state of ongoing and forthcoming trials of immune checkpoint blockade in HCC.

First draft submitted: 14 March 2016; Accepted for publication: 25 April 2016;

Published online: 27 June 2016
'Department of Medicine, The Hospital of the University of Pennsylvania, 3400 Spruce Street, Philadelphia, PA 19104, USA 2Departments of Medicine, Hematology \& Oncology, Abramson Cancer Center of the University of Pennsylvania, Perelman Center for Advanced Medicine, 6th Floor South Pavilion, 3400 Civic Center Boulevard, Philadelphia, PA 19104, USA

${ }^{3}$ Facultad de Medicina, Pontificia Universidad Javeriana, Cra. 7 No. 40-62, Hospital Universitario, San Ignacio, Bogota, Colombia *Author for correspondence: christopher.jensen@uphs.upenn.edu
Future Medicine 


\section{KEYWORDS}

- hepatocellular carcinoma

- immunotherapy

- sorafenib

\section{Background}

Hepatocellular carcinoma (HCC) is the second most common cause of cancer deaths worldwide and increasingly common in the USA, where the incidence of HCC nearly tripled from the 1980s [1-3]. HCC carries a poor prognosis, and available treatment modalities are limited. While local therapies offer possible cure, fewer options exist for advanced disease, as sorafenib is the only systemic agent [4]. Moreover, sorafenib's benefit is modest, increasing overall survival from 7.9 to 10.7 months [5]. Consequently, new treatment modalities are sorely needed. HCC is resistant to traditional chemotherapy regimens [6], and several targeted agents tested in Phase III trials (including sunitinib, brivanib, linifanib and erlotinib for firstline treatment and brivanib and everolimus after sorafenib failure), have all failed to show a benefit over sorafenib monotherapy [4]. Of note, while only marginal anti-tumor efficacy likely drove some of these negative results, other potential factors that contributed to negative findings include a limited understanding of drivers of disease progression (and thus limited ability to identify relevant patient subgroups), excess hepatic toxicity, and problems with study design. These issues have been recently and thoroughly reviewed by Llovet and colleagues $[4,7]$. Nevertheless, the disappointing results of these trials have contributed to interest in immune-based therapies.

The role for immunotherapy in HCC Immune-based therapies offer an appealing alternative, and there are theoretical reasons to hope they may prove an effective option. Spontaneous anti-tumor immune responses have been reported, and such responses may be disproportionately common in HCC compared with other tumors [8,9]. Moreover, HCC takes advantage of the relative immune tolerance of the liver, as well as checkpoints or brakes on the immune system, to avoid immune clearance.

A number of immunotherapeutic approaches have been investigated in HCC, including vaccines, adoptive cell transfer, cytokine-based treatments and immune checkpoint inhibitors, among others, though the data supporting the efficacy of many of these therapies is limited [10,11]. Of particular interest are immune checkpoint inhibitors, which have demonstrated benefit in other solid malignancies. They are of particular interest in light of the critical role immune checkpoints play in immunosuppression in the liver, both physiologically and in the setting of chronic inflammation and HCC.

\section{Immune checkpoints}

Activation of $T$ cells requires recognition of antigen by the T-cell receptor; however, this recognition alone is insufficient (Figure 1). Costimulatory signals must be provided by other receptors. Several receptor-ligand pairs have been identified, including CD28-CD80/CD86, CD137CD137L, CD27-CD70, OX40-OX40L and ICOS-B7RP1 [12]. Other surface receptors, or immune checkpoints, can instead provide coinhibitory signals that diminish the strength of T-cell responses. These immune checkpoints serve an important physiologic role in preventing autoimmunity; however, in the cancer microenvironment, the diminution of immune responses can contribute to tumor progression [13]. These coinhibitory receptor-ligand pairs include CTLA4-CD80/CD86, PD-1-PD-L1/PD-L2, Tim-3galectin 9, LAG3-MHC class I and II molecules, and KIR-MHC class I and II molecules $[10,13]$.

Recently, checkpoint inhibitors have demonstrated some efficacy in the treatment of multiple solid tumors. Ipilimumab, an anti-CTLA- 4 monoclonal antibody, was approved by the US FDA in 2011 for melanoma, and CTLA- 4 blockade has demonstrated some evidence of efficacy in other solid malignancies, though without improvement in overall outcomes. Similarly, PD-1 blockade has proven effective in Phase III trials for melanoma, along with evidence of anti-tumor activity in other solid tumors [14].

\section{CTLA-4 mechanism}

CTLA-4/CD152 was the first immune checkpoint to be therapeutically targeted [13]. This T-cell surface receptor, a member of the immunoglobulin superfamily, inhibits CD28, a T-cell costimulatory receptor (Figure 1). After antigen recognition by the T-cell receptor (TCR), signaling downstream of CD28 amplifies TCR signaling $[15,16]$. Upon TCR activation, CTLA-4 becomes localized to the cell membrane in direct proportion to the strength of TCR signaling, in a negative feedback mechanism [16-18]. There, CTLA- 4 competes with CD28 for its ligands, CD80 (B7.1) and CD86 (B7.2) [16,19]. CTLA-4 also mediates removal of $\mathrm{CD} 80$ and CD86 from the surface of antigen-presenting cells [20].

Along with this competitive mechanism, intracellular signaling downstream of CTLA- 4 contributes to T-cell inhibition. Though the 


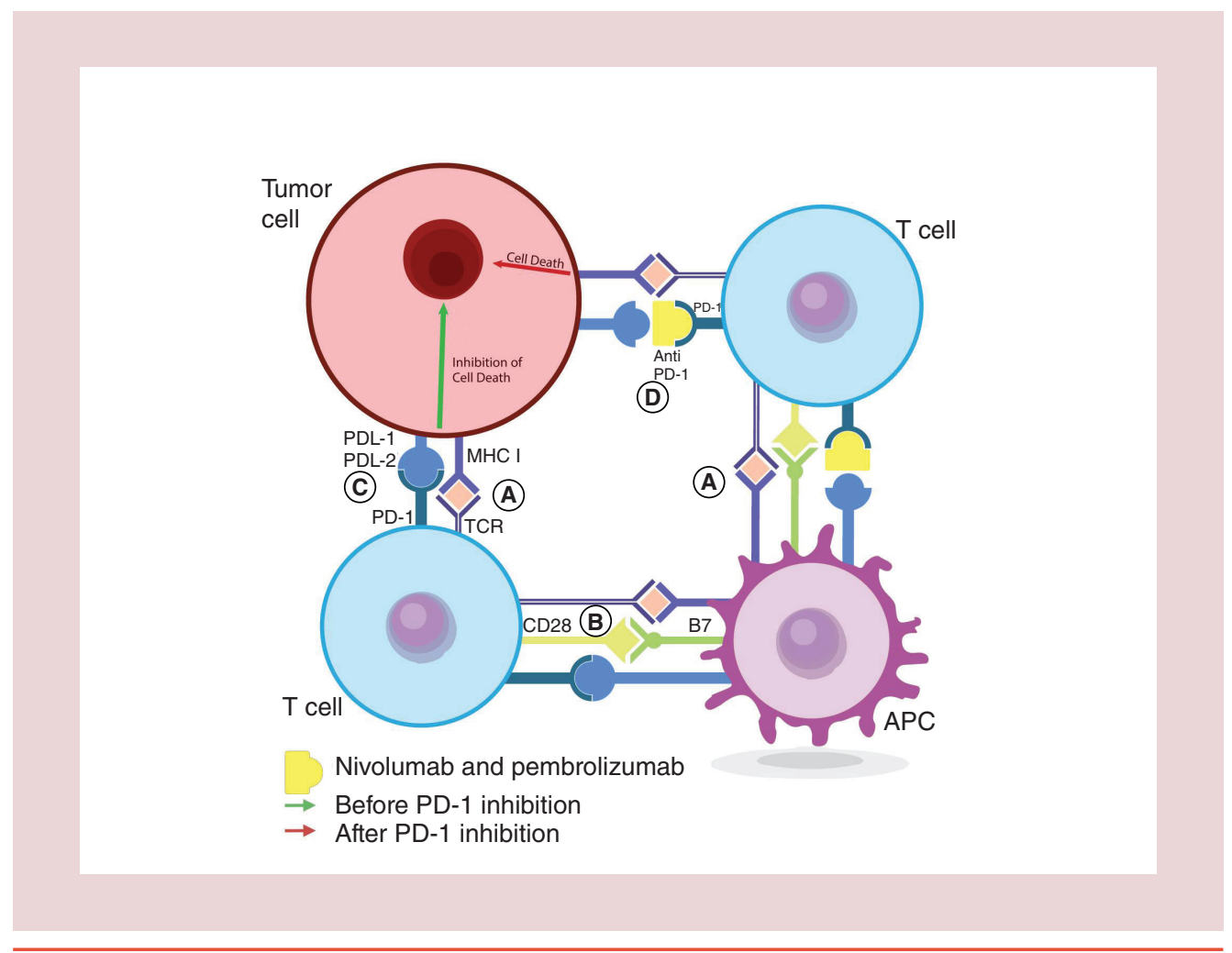

Figure 1. General mechanism of action of immune checkpoints and their inhibitors. (A) Activation of T cells requires recognition of antigen by the TCR in the context of an MHC molecule. MHC-II is involved in CD4 (helper) T-cell activation and is found on the surface of APCs, while MHC-I is involved in CD8 (cytotoxic) T-cell activation and is found on the surface of all nucleated cells. The intensity of the signaling downstream of this primary stimulus is modulated by several other cell surface receptors, two off which are shown here in simplified schematic. (B) CD28 is an example of a costimulatory receptor, which enhances stimulatory signaling in T cells upon binding to its ligand B7 on APCs. (C) By contrast, immune checkpoints, such as PD-1 and CTLA-4, are coinhibitory receptors, which diminish T-cell activation. Upon binding to its ligand (PD-L1), PD-1 inhibits T-cell activation and can promote T-cell apoptosis, thereby resulting in reduced T-cell mediated immune responses and, in the setting of malignancy, tumor cell survival via inhibition of cell death. (D) Immune checkpoint inhibitors (such as nivolumab and pembrolizumab) are monoclonal antibodies that block these coinhibitory receptors. Disruption of these inhibitory pathways can tip the intracellular signaling balance toward T-cell activation, thereby promoting T-cell-mediated killing of cancer cells. The broad mechanics of the CTLA-4 pathway and its inhibitors are similar to those diagramed above for PD-1, though CTLA-4 also directly binds to B7 (not shown), thereby also competitively inhibiting CD28. APC: Antigen-presenting cell; TCR: T-cell receptor.

exact mechanisms of CTLA-4 signaling remain unclear, inhibitory signals may be mediated by intracellular phosphatases, including SHP-1, SHP-2 and PP2A, which in turn dephosphorylate signaling kinases in the TCR pathway $[15,21,22]$. Functionally, CTLA-4 signaling drives increased T-cell motility and decreased contact time between $T$ cells and antigen-presenting cells [23].

CTLA-4 is also involved in the activity of $\mathrm{CD}^{+}{ }^{+} \mathrm{CD} 25^{+} \mathrm{Foxp}^{+}$regulatory $\mathrm{T}$ cells, which contribute to self tolerance and protection from autoimmunity [24-26]. Regulatory T cells constitutively express CTLA-4. The mechanisms by which CTLA-4 contributes to regulatory T-cell activity remain incompletely understood, but multiple signaling pathways involving both intracellular and extracellular activity by CTLA-4 have been implicated [27]

Physiologically, like other checkpoints, CTLA-4 provides protection against autoimmunity, and CTLA-4 knockout mice experience uncontrolled lymphoproliferation and autoimmunity $[25,28,29]$. 
PD-1 mechanism

PD-1 is another coinhibitory T-cell surface receptor in the immunoglobulin superfamily (Figure 1) [30-32]. Physiologically, PD-1 limits proinflammatory T-cell responses during infection, minimizing damage to healthy tissues and protecting against autoimmunity [13]. Activation of effector T cells stimulates PD-1 expression [32]. PD-1 then binds to its ligands and induces SHP-2 signaling, which inhibits kinases involved in T-cell activation, though other signaling pathways may also be involved [31,33]. These ligands include PD-L1 (B7-H1; CD274) and PD-L2 (CD273), which in addition to binding PD-1, contribute to other tolerogenic pathways $[31,34,35]$. PD-L1 and PD-L2 are upregulated in the setting of inflammation, in part due to the activity of IFN $-\gamma$, though the relevant signaling pathways have been incompletely elucidated [13].

As with CTLA-4, PD-1 is involved in the development of regulatory $\mathrm{T}$ cells [36,37]. High levels of PD-1 expression have been demonstrated in states of prolonged antigen exposure and are associated with anergy [38], and this state of relative immunosuppression is at least partially reversible via immune checkpoint blockade [39]. In addition, unlike CTLA-4, PD-1 is expressed on non T-cell leukocytes including $B$ cells and NK cells [13].

Immune evasion by HCC \& the role of immune checkpoints

Under physiologic conditions, the liver is an organ of relative immune tolerance, and checkpoint pathways play a key role in this process. The mechanisms of immune tolerance in the liver and relative immunosuppression in chronic inflammation and HCC have been recently reviewed $[10,11]$. Highlighted here are the roles of immune checkpoints in these processes.

\section{- Immune tolerance in the liver}

The liver is exposed to a myriad of food-borne and microbial antigens delivered from the gut, and the liver must be a relatively tolerance-inducing environment to prevent autoimmune-mediated damage. Hepatocytes and several nonparenchymal cell types mediate tolerance via the secretion of anti-inflammatory cytokines (including IL-10 and TGF- $\beta$ ), expression of PD-L1 and contributing to the development of regulatory $\mathrm{T}$ cells [40]. PD-L1 is expressed by hepatocytes [41], Kupffer cells [42], liver sinusoidal endothelial cells [43,44] and hepatic stellate cells [45]. As noted above, regulatory $\mathrm{T}$ cells constitutively express CTLA4 , and they have been linked to immune tolerance following liver transplant [46].

\section{- Immunosuppression of chronic inflammation}

Immunosuppression is increased by chronic inflammation, the background upon which HCC arises. As with physiologic tolerance, antiinflammatory cytokines, upregulated immune checkpoints and a relative abundance of regulatory $\mathrm{T}$ cells play key roles [11]. In this milieu, T cells may develop an 'exhausted' phenotype, characterized by hyporesponsiveness to antigen, increased checkpoint expression, and low levels of proinflammatory cytokines $[10,47]$.

Regarding checkpoints, chronic hepatitis drives increased PD-1 expression [42,48-50]. PD-L1 is also upregulated on multiple non-parenchymal cells in this setting, and the degree of upregulation is linked to the amount of inflammation $[42,48,51]$. CTLA-4 levels are also increased in patients with viral hepatitis [52]. Tim-3, a checkpoint that has not been therapeutically targeted, is linked to T-cell hypofunction in chronic hepatitis [53,54]. Regarding T-cell exhaustion, dual inhibition of PD-1 and CTLA-4 reverses the exhaustion of $\mathrm{CD}^{+}{ }^{+} \mathrm{T}$ cells from hepatitis-C-infected livers [39], and PD-1 blockade alone has a similar effect in the setting of hepatitis $\mathrm{B}[55,56]$.

\section{- HCC immune evasion \& the role of} immune checkpoints in this process In the setting of HCC, multiple immune derangements contribute to tumor survival and progression. These changes include ineffective antigen processing, increased numbers of regulatory $\mathrm{T}$ cells and other immunosuppressive leukocytes, diminished levels of $\mathrm{CD}^{+} \mathrm{T}$ cells, increased expression of checkpoints and deranged cytokine production [10,11]. Regarding immune checkpoints, the relationship between CTLA- 4 and regulatory $\mathrm{T}$ cells is again important, as increased regulatory T-cell levels have been repeatedly demonstrated in HCC [57-60]. In addition, decreased levels of costimulatory molecules and increased expression of checkpoints contribute to HCCrelated immunosuppression [11]. Several studies have linked checkpoint pathways to immune dysregulation in HCC, including CTLA-4 [61,62], PD-1-PD-L1 [50,63-67], LAG-3 [68], KIR [69,70] and the Tim-3-galectin-9 [71,72] pathways.

In tumor microenvironments, PD-1 induces effector T-cell apoptosis and mediates immune 
evasion, a pathway tumor cells utilize via expression of PD-L1 or PD-L2 [30,73-75], a welldescribed phenomenon in other malignancies (particularly melanoma) [76]. In HCC, increased PD-1 signaling has clinically relevant implications for patients $[11,30]$. PD-1 upregulation has been described on effector T cells in HCC [63,64], and PD-L1 is highly expressed on both HCC tumor cells and local stromal cells $[63,67,77,78]$. In one prospective study, higher levels of PD-1positive effector $\mathrm{T}$ cells correlated with progression of disease following resection [64]. In a separate retrospective cohort study, increased local PD-L1 levels were associated with increased postoperative recurrence [78]. Higher levels of circulating PD-1 and PD-L1 on flow cytometry have also been associated with shorter survival after cryoablation [79]. A more recent retrospective study demonstrated a trend toward improved overall survival after resection among patients whose tumors had lower levels of PD-L1, though this effect was not statistically significant [80].

Unfortunately, less data from preclinical and translational studies exist regarding the mechanistic role of CTLA-4 in HCC [30]. However, high levels of CTLA-4 expression on hepatic dendritic cells have been linked to inhibition of T-cell proliferation and to T-cell apoptosis in HCC [61], and high levels of CTLA-4 expression by regulatory $\mathrm{T}$ cells correlate with decreased production of cytotoxic enzymes by effector $\mathrm{T}$ cells in HCC [57]. With regard to the CTLA-4 pathway's role in regulatory T-cell development, increased levels of regulatory $\mathrm{T}$ cells have been associated with both decreased levels of effector $\mathrm{T}$ cells in the tumor microenvironment $[59,60]$ and, more clinically relevant, with decreased overall survival in patients with HCC [81,82].

Trials of CTLA-4 \& PD-1 blockade in HCC

Several clinical trials of immune checkpoint inhibitors in HCC are ongoing, and the results of some of these trials have already been reported (Table 1). Results of a Phase II trial of tremelimumab, a fully human anti-CTLA- 4 monoclonal antibody were published in 2013 (ClinicalTrials.gov: NCT01008358) [83]. Patients with unresectable HCC and chronic hepatitis C received tremelimumab monotherapy until progression of disease or severe toxicity developed. Treatment was generally well tolerated, with few patients experiencing disabling adverse events. The most common adverse events were rash, and $45 \%$ of patients experienced a grade 3 or 4 transaminase elevation without decline in liver function. Among 20 patients treated, there was a $76.4 \%$ disease control rate, consisting of zero complete responses, three partial responses, and ten instances of stable disease. The median time to progression was 6.5 months. An HCV virologic response was also noted, with 12 patients experiencing a marked reduction in HCV viral load and three experiencing temporary complete virologic responses.

More recently, tremelimumab has been studied with local ablative therapies (radiofrequency ablation or chemoembolization) for patients with advanced HCC who were intolerant or refractory to sorafenib (ClinicalTrials. gov: NCT01853618) [84]. This study began in 2013 and is ongoing, though preliminary results were presented at the 2015 American Society of Clinical Oncology (ASCO) annual meeting. Of 20 patients enrolled, ten had lesions that could be evaluated outside of their TACE/RFA target. Of these, four had partial responses. Also, a marked virologic response was again noted, and five of the seven patients with detectable viral loads had reductions with treatment.

Regarding PD-1 therapies, a Phase I/II trial of the anti-PD-1 monoclonal antibody pidilizumab (CT-011) began in 2009; however, the trial was terminated in 2012 due to slow accrual (ClinicalTrials.gov: NCT00966251). In a subsequent Phase I/II trial begun in 2013, MEDI4736, a fully human anti-PD-L1 monoclonal antibody, is being evaluated in multiple advanced cancers, including HCC (ClinicalTrials.gov: NCT01938612) [85]. Only initial data have been reported, which included a relatively favorable safety profile, and further recruitment is ongoing.

Preliminary results of a Phase I/II trial of nivolumab, a fully human anti-PD-1 monoclonal antibody, were reported at the 2015 ASCO annual meeting (ClinicalTrials.gov: NCT01658878) [86]. This study, which began in 2012 and included patients both with and without viral hepatitis, recruited patients who had failed sorafenib (due to progression of disease, intolerance, or refusal). Of 41 patients, 39 had an evaluable response. Responses included two complete responses and seven objective partial responses. Overall survival was $72 \%$ at 6 months and $62 \%$ at 12 months. Of note, $68 \%$ of patients had received prior sorafenib therapy, and responses were seen in both patients with and without viral hepatitis. Grade 3-4 adverse events included transaminase and lipase elevations along with individual instances 


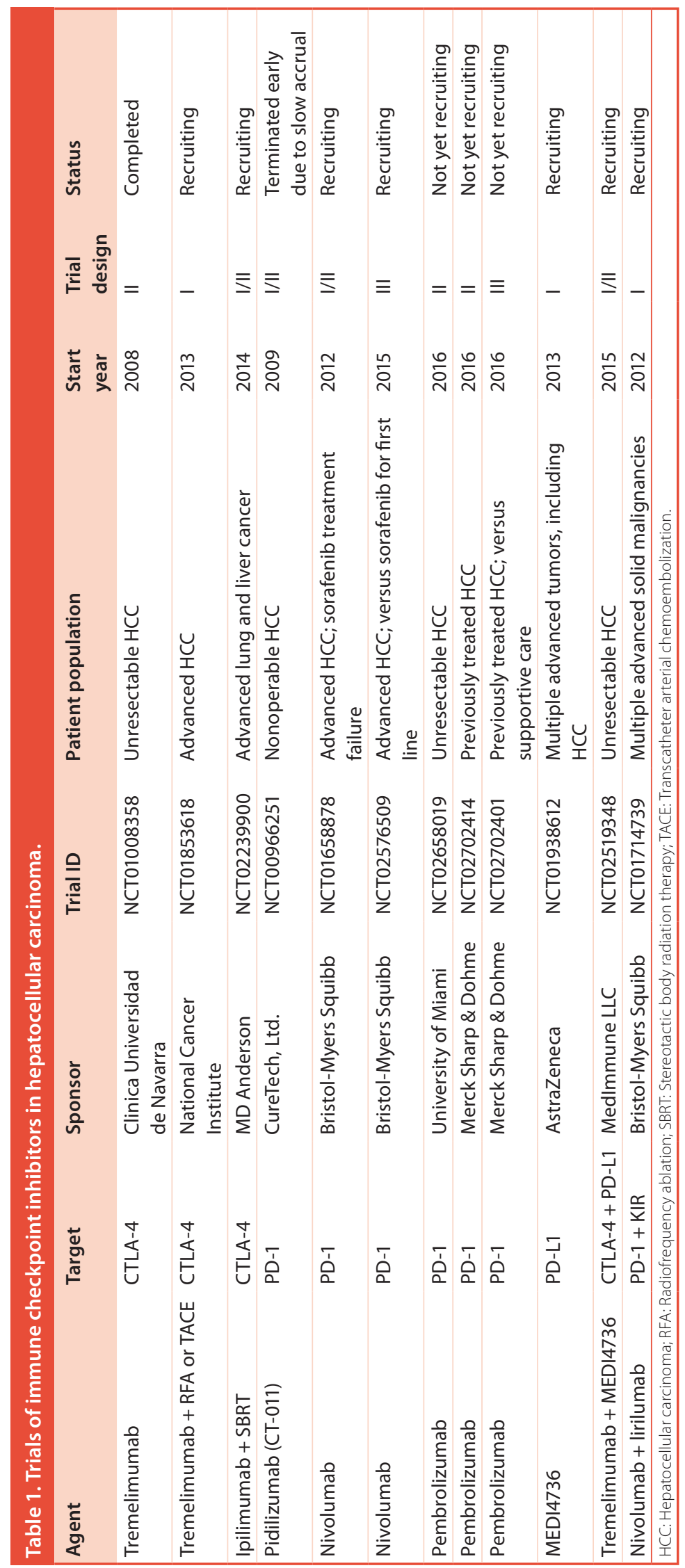

of anemia and fatigue, and the overall safety profile was similar to that seen in prior studies of nivolumab.

Following these data, a recently-initiated Phase III trial (CheckMate 459) will compare nivolumab to sorafenib for first-line treatment of advanced HCC (ClinicalTrials.gov: NCT02576509). Given the limited systemic therapies for advanced HCC, as well as the results reported by El-Khoueiry and colleagues of noteworthy response rates among patients with prior sorafenib treatment, the initiation of this trial is a noteworthy development. Finally, several related upcoming trials will assess the effectiveness of pembrolizumab, a PD-1 antagonist previously unused in HCC (Table 1). Such further research is certainly needed to validate the effectiveness of checkpoint inhibitors; however, these trials offer the potential for new and sorely needed treatment modalities for advanced HCC.

\section{Complications of CTLA-4 \& PD-1 blockade}

Despite these promising results, checkpoint inhibitor therapy may be complicated by immunerelated adverse events, as the immune responses these agents promote are not tumor specific. As reviewed by Postow and colleagues, skin, liver, gastrointestinal and endocrine immune-mediated toxicities have been described along with other less common side effects, with skin findings being most common [14]. Such manifestations may necessitate immunosuppression with steroids and other agents, though evidence for the management of these complications is limited. Of particular note are the colitis described in a minority of patients receiving CTLA- 4 inhibitors, which has resulted in a small number of treatmentrelated deaths, and the grade 3-4 transaminase elevations described with both CTLA- 4 and PD-1 blockade. This latter hepatic complication, which was seen in the trials of tremelimumab and nivolumab discussed above, is generally asymptomatic but may require treatment with systemic steroids or mycophenolate in refractory cases [14]. Two issues regarding these adverse events are worthy of particular note. First, most of these adverse events were described in patients with malignancies other than HCC, and toxicity profiles may differ for patients with HCC, particularly given the prevalence of cirrhosis in this population. Second, the need to control the more severe of these adverse events with immunosuppression may diminish the therapeutic antitumor immune response of checkpoint inhibitors. 
Beyond CTLA-4 \& PD-1 blockade monotherapy

In addition to anti-CTLA- 4 and PD-1 monotherapy, combination therapy stands as a critical direction for further research [10]. The multiple mechanisms of HCC immune evasion suggest that multiple immunotherapies may be necessary to drive effective immune responses against HCC. For instance, dual inhibition of PD-1 and CTLA- 4 is required to reverse T-cell exhaustion in chronic hepatitis $C[39]$. Such a trial of combined PD-1/CTLA-4 blockade with nivolumab and ipilimumab was conducted among patients with untreated melanoma, with an improvement in median progression-free survival to 11.5 months with combined therapy versus 2.9 months for ipilimumab and 6.9 months for nivolumab. However, this improvement came with an increased rate of grade 3-4 toxicities, which affected $55.0 \%$ of the combined-therapy group compared with $16.3 \%$ of those receiving nivolumab and $27.3 \%$ of those receiving ipilimumab [87].

For HCC, no similar trials have been reported. Of note, the protocol of the Phase I/II study reported at ASCO by El-Khoueiry and colleagues was modified in November 2015 to include a nivolumab/ipilimumab combination arm (ClinicalTrials.gov: NCT01658878). Two other trials of combined checkpoint inhibitors are ongoing, including a study of tremelimumab with MEDI4736 (a PD-L1 antagonist) for patients with unresectable HCC begun in 2015 (ClinicalTrials.gov: NCT02519348) and a Phase I trial of lirilumab (an anti-KIR monoclonal antibody) with nivolumab for multiple advanced malignancies, including HCC (ClinicalTrials.gov: NCT01714739). Moreover, this last trial highlights an additional promising area of further research: the development of inhibitors for other immune checkpoints beyond PD-1-PD-L1 and CTLA-4. The KIRMHC [69,70], LAG-3-MHC [68] and Tim-3galectin-9 checkpoint pathways [71,72] have all been linked to T-cell dysfunction in HCC.

Besides multiple checkpoint inhibition, the combination of checkpoint blockade with sorafenib will be an important area of future research as sorafenib has been linked to immunomodulatory effects, including disruption of T-cell proliferation and function [88]. There is also preclinical data supporting a synergistic effect between sorafenib and checkpoint inhibitors in other malignancies [89]. More recently, Chen and colleagues demonstrated that sorafenib induces local hypoxia in the tumor microenvironment, which in turn drives immunosuppression characterized by increased expression of PD-L1 and an accumulation of regulatory $\mathrm{T}$ cells [90]. The recruitment of immunosuppressive cells was partially mediated by upregulation of SDF1 $1 \alpha$, and the addition of an inhibitor of the SDF1 $\alpha$ receptor to sorafenib therapy prevented this immunosuppressive effect and enhanced the antineoplastic effects of sorafenib, as measured by tumor growth and metastasis in a murine model. Furthermore, addition of a PD-1 antagonist to this cocktail further enhanced its inhibition of HCC growth. To the best of our knowledge, no trials combining sorafenib and checkpoint inhibitors are ongoing; however, this strategy presents a promising avenue for future research.

Like checkpoint inhibitors, a similar rational underpins treatment with agonists of costimulatory pathways. Such agents have demonstrated benefit in murine models by targeting the OX40 [91] and CD137 pathways [91,92]. A Phase I trial of an OX40 agonist has been initiated for patients with several advanced malignancies, including HCC (ClinicalTrials.gov: NCT02315066). Regarding the CD137 pathway, a Phase I trial of urelumab (a CD137 agonist) combined with nivolumab is also recruiting patients with multiple advanced solid malignancies (ClinicalTrials.gov: NCT02534506).

Conclusion \& future perspective

As noted above, the disease burden of HCC is substantial. The benefit of sorafenib monotherapy is limited, and other effective systemic therapies have not yet been identified. In this light, the preliminary results of checkpoint inhibitor trials offer a promising path forward. While we acknowledge that issues besides limited anti-tumor activity alone have contributed to negative clinical trials in HCC, given the failure of other molecularly targeted agents and more traditional chemotherapy, immune checkpoint blockade stands as one of the most promising potential therapies for HCC. Consequently, the initiation of a Phase III trial comparing nivolumab to sorafenib in the first-line setting is an important development. However, in light of the multiple mechanisms by which HCC evades the immune system, as well as possible immunosuppressive effects of sorafenib in the tumor microenvironment, additional research into combination therapy is warranted.

While this review has focused on the pressing need for more effective systemic therapies 
for advanced HCC, an additional avenue for further research is the combination of locoregional therapies with checkpoint blockade for earlier-stage disease. Of note, one such study is ongoing, including patients treated with radiofrequency ablation or chemoembolization and tremelimumab (ClinicalTrials.gov: NCT01853618).

Finally, given variable responses to checkpoint blockade seen in HCC and other malignancies, the identification of biomarkers predictive of response would be desirable. Unfortunately, no clear markers of response to anti-CTLA-4 therapy have been identified for HCC [10,11], and while local PD-L1 expression has been somewhat correlated with response rates for anti-PD-1 antibodies, patients without significant PD-L1 expression have nonetheless been noted to have dramatic responses to treatment [14]. Consequently, in addition to trials of new combinations of immune-active agents, further research is needed to clarify what factors drive responses to checkpoint inhibitor therapy.

Financial \& competing interests disclosure A Loaiza-Bonilla declares consulting fees from Massive Bio, Inc, Bayer, Genentech and Merrimack. The authors have no other relevant affiliations or financial involvement with any organization or entity with a financial interest in or financial conflict with the subject matter or materials discussed in the manuscript apart from those disclosed.

No writing assistance was utilized in the production of this manuscript.

\section{References}

Papers of special note have been highlighted as:

- of interest; $\bullet \bullet$ of considerable interest

1 Ferlay J, Soerjomataram I, Ervik M et al. GLOBOCAN 2012 v1.0, Cancer Incidence and Mortality Worldwide: IARC CancerBase No. 11. 2016 (02/15/2016), (2013).

2 El-Serag HB, Kanwal F. Epidemiology of hepatocellular carcinoma in the United States: where are we? Where do we go? Hepatology 60(5), 1767-1775 (2014).

3 Centers for Disease Control and Prevention. Hepatocellular carcinoma - United States, 2001-2006. MMWR Morb. Mortal. Wkly Rep. 59(17), 517-520 (2010).

4 Llovet JM, Villanueva A, Lachenmayer A, Finn RS. Advances in targeted therapies for hepatocellular carcinoma in the genomic era. Nat. Rev. Clin. Oncol. 12(8), 436 (2015).

5 Llovet JM, Ricci S, Mazzaferro V et al. Sorafenib in advanced hepatocellular carcinoma. N. Engl. J. Med. 359(4), 378-390 (2008).

- Sorafenib, whose efficacy was demonstrated in a Phase III trial in 2008, remains the only currently approved systemic agent for advanced hepatocellular carcinoma (HCC).

6 Qin S, Bai Y, Lim HY et al. Randomized, multicenter, open-label study of oxaliplatin plus fluorouracil/leucovorin versus doxorubicin as palliative chemotherapy in patients with advanced hepatocellular carcinoma from Asia. J. Clin. Oncol. 31(28), 3501-3508 (2013).

7 Llovet JM, Hernandez-Gea V. Hepatocellular carcinoma: reasons for Phase III failure and novel perspectives on trial design. Clin. Cancer Res. 20(8), 2072-2079 (2014).
8 Greten TF, Wang XW, Korangy F. Current concepts of immune based treatments for patients with HCC: from basic science to novel treatment approaches. Gut 64(5), 842-848 (2015).

9 Huz JI, Melis M, Sarpel U. Spontaneous regression of hepatocellular carcinoma is most often associated with tumour hypoxia or a systemic inflammatory response. $H P B$ (Oxford) 14(8), 500-505 (2012).

10 Harding JJ, El Dika I, Abou-Alfa GK. Immunotherapy in hepatocellular carcinoma: primed to make a difference? Cancer 122(3), 367-377 (2016).

11 Makarova-Rusher OV, Medina-Echeverz J, Duffy AG, Greten TF. The yin and yang of evasion and immune activation in HCC. J. Hepatol. 62 (6), 1420-1429 (2015).

12 Chen L, Flies DB. Molecular mechanisms of $\mathrm{T}$ cell co-stimulation and co-inhibition. Nat. Rev. Immunol. 13(4), 227-242 (2013).

13 Pardoll DM. The blockade of immune checkpoints in cancer immunotherapy. Nat. Rev. Cancer 12(4), 252-264 (2012).

14 Postow MA, Callahan MK, Wolchok JD. Immune checkpoint blockade in cancer therapy. J. Clin. Oncol. 33(17), 1974-1982 (2015).

15 Rudd CE, Taylor A, Schneider H. CD28 and CTLA- 4 coreceptor expression and signal transduction. Immunol. Rev. 229(1), 12-26 (2009).

16 Linsley PS, Brady W, Urnes M, Grosmaire LS, Damle NK, Ledbetter JA. CTLA-4 is a second receptor for the $\mathrm{B}$ cell activation antigen B7. J. Exp. Med. 174(3), 561-569 (1991).
- The original description of CTLA-4.

17 Egen JG, Allison JP. Cytotoxic T lymphocyte antigen- 4 accumulation in the immunological synapse is regulated by TCR signal strength. Immunity 16(1), 23-35 (2002).

18 Walunas TL, Lenschow DJ, Bakker CY et al. CTLA- 4 can function as a negative regulator of $\mathrm{T}$ cell activation. Immunity 1(5), 405-413 (1994).

19 Collins AV, Brodie DW, Gilbert RJ et al. The interaction properties of costimulatory molecules revisited. Immunity 17(2), 201-210 (2002).

20 Qureshi OS, Zheng Y, Nakamura K et al. Trans-endocytosis of CD80 and CD86: a molecular basis for the cell-extrinsic function of CTLA-4. Science 332(6029), 600-603 (2011).

21 Buchbinder E, Hodi FS. Cytotoxic tlymphocyte antigen- 4 and immune checkpoint blockade. J. Clin. Invest. 125(9), 3377-3383 (2015).

22 Parry RV, Chemnitz JM, Frauwirth KA et al. CTLA-4 and PD-1 receptors inhibit T-cell activation by distinct mechanisms. Mol. Cell. Biol. 25(21), 9543-9553 (2005).

23 Schneider H, Downey J, Smith A et al. Reversal of the TCR stop signal by CTLA-4. Science 313(5795), 1972-1975 (2006).

24 Peggs KS, Quezada SA, Chambers CA, Korman AJ, Allison JP. Blockade of CTLA-4 on both effector and regulatory $\mathrm{T}$ cell compartments contributes to the antitumor activity of anti-CTLA-4 antibodies. J. Exp. Med. 206(8), 1717-1725 (2009).

25 Wing K, Onishi Y, Prieto-Martin P et al. CTLA-4 control over Foxp $3^{+}$regulatory T cell function. Science 322(5899), 271-275 (2008). 
26 Takahashi T, Tagami T, Yamazaki S et al. Immunologic self-tolerance maintained by $\mathrm{CD} 25\left(^{+}\right) \mathrm{CD} 4\left(^{+}\right)$regulatory $\mathrm{T}$ cells constitutively expressing cytotoxic $\mathrm{T}$ lymphocyteassociated antigen 4. J. Exp. Med. 192(2), 303-310 (2000).

27 Tai X, Van Laethem F, Pobezinsky L et al. Basis of CTLA- 4 function in regulatory and conventional CD4(+) T cells. Blood $119(22)$, 5155-5163 (2012).

28 Tivol EA, Boyd SD, Mckeon S et al. CTLA-4-Ig prevents lymphoproliferation and fatal multiorgan tissue destruction in CTLA-4-deficient mice. J. Immunol. 158(11), 5091-5094 (1997).

29 Waterhouse R, Penninger JM, Timms E et al. Lymphoproliferative disorders with early lethality in mice deficient in Ctla-4. Science 270 (5238), 985-988 (1995).

30 Hato T, Goyal L, Greten TF, Duda DG, Zhu AX. Immune checkpoint blockade in hepatocellular carcinoma: current progress and future directions. Hepatology 60(5), 1776-1782 (2014).

31 Freeman GJ, Long AJ, Iwai Y et al. Engagement of the PD-1 immunoinhibitory receptor by a novel B7 family member leads to negative regulation of lymphocyte activation. J. Exp. Med. 192(7), 1027-1034 (2000).

32 Ishida Y, Agata Y, Shibahara K, Honjo T. Induced expression of PD-1, a novel member of the immunoglobulin gene superfamily, upon programmed cell death. EMBO J. 11(11), 3887-3895 (1992).

- The original description of PD-1.

33 Okazaki T, Maeda A, Nishimura H, Kurosaki T, Honjo T. PD-1 immunoreceptor inhibits $B$ cell receptor-mediated signaling by recruiting src homology 2-domain-containing tyrosine phosphatase 2 to phosphotyrosine. Proc. Natl Acad. Sci. USA 98(24), 13866-13871 (2001).

34 Latchman Y, Wood CR, Chernova T et al. PD-L2 is a second ligand for PD-1 and inhibits T cell activation. Nat. Immunol. 2(3), 261-268 (2001).

35 Park JJ, Omiya R, Matsumura Y et al. $\mathrm{B} 7-\mathrm{H} 1 / \mathrm{CD} 80$ interaction is required for the induction and maintenance of peripheral T-cell tolerance. Blood 116(8), 1291-1298 (2010).

36 Francisco LM, Salinas VH, Brown KE et al. PD-L1 regulates the development, maintenance, and function of induced regulatory T cells. J. Exp. Med. 206(13), 3015-3029 (2009).

37 Nikolova M, Lelievre JD, Carriere M, Bensussan A, Levy Y. Regulatory T cells differentially modulate the maturation and apoptosis of human $\mathrm{CD} 8^{+} \mathrm{T}$-cell subsets. Blood 113(19), 4556-4565 (2009).

38

Barber DL, Wherry EJ, Masopust D et al. Restoring function in exhausted CD8 T cells during chronic viral infection. Nature 439 (7077), 682-687 (2006).

39 Nakamoto N, Cho H, Shaked A et al. Synergistic reversal of intrahepatic HCVspecific CD8 $\mathrm{T}$ cell exhaustion by combined PD-1/CTLA-4 blockade. PLoS Pathog. 5(2), e1000313 (2009).

40 Tiegs G, Lohse AW. Immune tolerance: what is unique about the liver. J. Autoimmun. 34(1), 1-6 (2010).

41 Wahl C, Bochtler P, Chen L, Schirmbeck R, Reimann J. B7-H1 on hepatocytes facilitates priming of specific CD8 T cells but limits the specific recall of primed responses. Gastroenterology 135(3), 980-988 (2008).

42 Kassel R, Cruise MW, Iezzoni JC, Taylor NA, Pruett TL, Hahn YS. Chronically inflamed livers up-regulate expression of inhibitory B7 family members. Hepatology 50(5), 1625-1637 (2009).

43 Carambia A, Frenzel C, Bruns OT et al. Inhibition of inflammatory $\mathrm{CD} 4 \mathrm{~T}$ cell activity by murine liver sinusoidal endothelial cells. J. Hepatol. 58(1), 112-118 (2013).

44 Diehl L, Schurich A, Grochtmann R, Hegenbarth S, Chen L, Knolle PA. Tolerogenic maturation of liver sinusoidal endothelial cells promotes B7-homolog 1-dependent $\mathrm{CD}^{+}{ }^{+} \mathrm{T}$ cell tolerance. Hepatology 47(1), 296-305 (2008).

$45 \mathrm{Yu} \mathrm{MC}$, Chen $\mathrm{CH}$, Liang $\mathrm{X}$ et al. Inhibition of T-cell responses by hepatic stellate cells via B7-H1-mediated T-cell apoptosis in mice. Hepatology 40 (6), 1312-1321 (2004).

46 Li W, Kuhr CS, Zheng XX et al. New insights into mechanisms of spontaneous liver transplant tolerance: the role of Foxp3expressing $\mathrm{CD} 25^{+} \mathrm{CD} 4{ }^{+}$regulatory $\mathrm{T}$ cells. Am. J. Transplant. 8(8), 1639-1651 (2008).

47 Jiang Y, Li Y, Zhu B. T-cell exhaustion in the tumor microenvironment. Cell Death Dis. 6, e1792 (2015).

48 Chen J, Wang XM, Wu XJ et al. Intrahepatic levels of PD-1/PD-L correlate with liver inflammation in chronic hepatitis B. Inflamm. Res. 60 (1), 47-53 (2011).

49 Peng G, Li S, Wu W, Tan X, Chen Y, Chen Z. PD-1 upregulation is associated with HBVspecific $T$ cell dysfunction in chronic hepatitis B patients. Mol. Immunol. 45(4), 963-970 (2008).

50 Nakamoto N, Kaplan DE, Coleclough J et al. Functional restoration of HCV-specific CD8
T cells by PD- 1 blockade is defined by PD-1 expression and compartmentalization. Gastroenterology 134(7), 1927-1937, 1937.e1-e2 (2008).

51 Mühlbauer M, Fleck M, Schütz C et al. PD-L1 is induced in hepatocytes by viral infection and by interferon-alpha and -gamma and mediates T cell apoptosis. J. Hepatol. 45(4), 520-528 (2006).

52 Schurich A, Khanna P, Lopes AR et al. Role of the coinhibitory receptor cytotoxic T lymphocyte antigen- 4 on apoptosis-prone CD8 $T$ cells in persistent hepatitis B virus infection. Hepatology 53(5), 1494-1503 (2011).

53 Moorman JP, Wang JM, Zhang Y et al. Tim-3 pathway controls regulatory and effector $\mathrm{T}$ cell balance during hepatitis $\mathrm{C}$ virus infection. J. Immunol. 189(2), 755-766 (2012).

54 Nebbia G, Peppa D, Schurich A et al. Upregulation of the Tim-3/galectin-9 pathway of $\mathrm{T}$ cell exhaustion in chronic hepatitis $\mathrm{B}$ virus infection. PLoS ONE 7(10), e47648 (2012).

55 Bengsch B, Martin B, Thimme R. Restoration of $\mathrm{HBV}$-specific $\mathrm{CD} 8^{+} \mathrm{T}$ cell function by PD-1 blockade in inactive carrier patients is linked to $\mathrm{T}$ cell differentiation. J. Hepatol. 61(6), 1212-1219 (2014).

56 Fisicaro P, Valdatta C, Massari M et al. Antiviral intrahepatic T-cell responses can be restored by blocking programmed death-1 pathway in chronic hepatitis B. Gastroenterology 138(2), 682-693, 693, e681-684 (2010).

57 Kalathil S, Lugade AA, Miller A, Iyer R, Thanavala Y. Higher frequencies of $\operatorname{GARP}\left({ }^{+}\right)$ CTLA- $4\left(^{+}\right)$Foxp $3\left(^{+}\right)$T regulatory cells and myeloid-derived suppressor cells in hepatocellular carcinoma patients are associated with impaired T-cell functionality. Cancer Res. 73(8), 2435-2444 (2013).

58 Pedroza-Gonzalez A, Verhoef C, Ijzermans JN et al. Activated tumor-infiltrating $\mathrm{CD}_{4}{ }^{+}$ regulatory $\mathrm{T}$ cells restrain antitumor immunity in patients with primary or metastatic liver cancer. Hepatology 57(1), 183-194 (2013).

59 Ormandy LA, Hillemann T, Wedemeyer $\mathrm{H}$, Manns MP, Greten TF, Korangy F. Increased populations of regulatory $\mathrm{T}$ cells in peripheral blood of patients with hepatocellular carcinoma. Cancer Res. 65(6), 2457-2464 (2005).

60 Unitt E, Rushbrook SM, Marshall A et al. Compromised lymphocytes infiltrate hepatocellular carcinoma: the role of T-regulatory cells. Hepatology 41(4), 722-730 (2005). 
61 Han Y, Chen Z, Yang Y et al. Human CD14 CTLA $-4^{+}$regulatory dendritic cells suppress T-cell response by cytotoxic T-lymphocyte antigen-4-dependent IL-10 and indoleamine-2,3-dioxygenase production in hepatocellular carcinoma. Hepatology 59(2), 567-579 (2014).

62 Mizukoshi E, Nakamoto Y, Arai K et al. Comparative analysis of various tumor-associated antigen-specific t-cell responses in patients with hepatocellular carcinoma. Hepatology 53(4), 1206-1216 (2011).

63 Wu K, Kryczek I, Chen L, Zou W, Welling TH. Kupffer cell suppression of CD8 ${ }^{+} \mathrm{T}$ cells in human hepatocellular carcinoma is mediated by $\mathrm{B} 7-\mathrm{H} 1 /$ programmed death-1 interactions. Cancer Res. 69 (20), 8067-8075 (2009).

64 Shi F, Shi M, Zeng Z et al. PD-1 and PD-L1 upregulation promotes $\mathrm{CD} 8\left(^{+}\right)$T-cell apoptosis and postoperative recurrence in hepatocellular carcinoma patients. Int. J. Cancer 128(4), 887-896 (2011).

65 He G, Zhang H, Zhou J et al. Peritumoural neutrophils negatively regulate adaptive immunity via the PD-L1/PD-1 signalling pathway in hepatocellular carcinoma. J. Exp. Clin. Cancer Res. 34141 (2015).

66 Geng L, Deng J, Jiang G et al. B7-H1 up-regulated expression in human hepatocellular carcinoma tissue: correlation with tumor interleukin-10 levels. Hepatogastroenterology 58(107-108), 960-964 (2011).

67 Kuang DM, Zhao Q, Peng C et al. Activated monocytes in peritumoral stroma of hepatocellular carcinoma foster immune privilege and disease progression through PD-L1. J. Exp. Med. 206(6), 1327-1337 (2009).

68 Li FJ, Zhang Y, Jin GX, Yao L, Wu DQ. Expression of LAG-3 is coincident with the impaired effector function of HBV-specific CD8 $\left(^{+}\right) \mathrm{T}$ cell in HCC patients. Immunol. Lett. 150(1-2), 116-122 (2013).

69 Pan N, Qiu J, Sun H et al. Combination of human leukocyte antigen and killer cell immunoglobulin-like receptor genetic background influences the onset age of hepatocellular carcinoma in male patients with hepatitis B virus infection. Clin. Dev. Immunol. 2013, 874514 (2013).

70 Cariani E, Missale G. KIR/HLA immunogenetic background influences the evolution of hepatocellular carcinoma. Oncoimmunology 2(12), e26622 (2013).

71 Yan W, Liu X, Ma H et al. Tim-3 fosters HCC development by enhancing TGF-betamediated alternative activation of macrophages. Gut 64(10), 1593-1604 (2015).
72 Li H, Wu K, Tao K et al. Tim-3/galectin-9 signaling pathway mediates T-cell dysfunction and predicts poor prognosis in patients with hepatitis B virus-associated hepatocellular carcinoma. Hepatology 56(4), 1342-1351 (2012).

73 Zou W, Chen L. Inhibitory B7-family molecules in the tumour microenvironment. Nat. Rev. Immunol. 8(6), 467-477 (2008).

74 Iwai Y, Ishida M, Tanaka Y, Okazaki T, Honjo T, Minato N. Involvement of PD-L1 on tumor cells in the escape from host immune system and tumor immunotherapy by PD-L1 blockade. Proc. Natl Acad. Sci. USA 99(19), 12293-12297 (2002).

75 Dong H, Strome SE, Salomao DR et al. Tumor-associated B7-H1 promotes T-cell apoptosis: a potential mechanism of immune evasion. Nat. Med. 8(8), 793-800 (2002).

76 Taube JM, Anders RA, Young GD et al. Colocalization of inflammatory response with B7-h1 expression in human melanocytic lesions supports an adaptive resistance mechanism of immune escape. Sci. Transl. Med. 4(127), 127ra137 (2012).

77 Wang BJ, Bao JJ, Wang JZ et al. Immunostaining of PD-1/PD-Ls in liver tissues of patients with hepatitis and hepatocellular carcinoma. World J. Gastroenterol. 17(28), 3322-3329 (2011).

78 Gao Q, Wang XY, Qiu SJ et al. Overexpression of PD-L1 significantly associates with tumor aggressiveness and postoperative recurrence in human hepatocellular carcinoma. Clin. Cancer Res. 15(3), 971-979 (2009).

79 Zeng Z, Shi F, Zhou L et al. Upregulation of circulating PD-L1/PD-1 is associated with poor post-cryoablation prognosis in patients with HBV-related hepatocellular carcinoma. PLoS ONE 6(9), e23621 (2011).

80 Umemoto Y, Okano S, Matsumoto Y et al. Prognostic impact of programmed cell death 1 ligand 1 expression in human leukocyte antigen class I-positive hepatocellular carcinoma after curative hepatectomy. J. Gastroenterol. 50(1), 65-75 (2015).

81 Gao Q, Qiu SJ, Fan J et al. Intratumoral balance of regulatory and cytotoxic T cells is associated with prognosis of hepatocellular carcinoma after resection. J. Clin. Oncol. 25(18), 2586-2593 (2007).

$82 \mathrm{Fu}$ J, Xu D, Liu Z et al. Increased regulatory $\mathrm{T}$ cells correlate with CD8 T-cell impairment and poor survival in hepatocellular carcinoma patients. Gastroenterology 132(7), 2328-2339 (2007).
83 Sangro B, Gomez-Martin C, De La Mata M et al. A clinical trial of CTLA-4 blockade with tremelimumab in patients with hepatocellular carcinoma and chronic hepatitis C. J. Hepatol. 59(1), 81-88 (2013).

- Sangro and colleagues described the first completed trial of an immune checkpoint inhibitor in HCC.

84 Duffy AG, Makarova-Rusher OV, Kerkar SP et al. A pilot study of tremelimumab - a monoclonal antibody against CTLA-4 - in combination with either trans catheter arterial chemoembolization (TACE) or radiofrequency ablation (RFA) in patients with hepatocellular carcinoma (HCC). Presented at: 51st Annual Meeting of American Society OfClinical Oncology. Chicago, IL, USA, 29 May-2 June 2015.

85 Segal NH, Hamid O, Hwu W et al. A Phase I multi-arm dose-expansion study of the anti-programmed cell death-ligand-1 (PD-L1) antibody MEDI4736: preliminary data. Ann. Oncol. 25(Suppl. 4), iv365 (2014).

86 El-Khoueiry AB, Melero I, Crocenzi TS et al. Phase I/II safety and antitumor activity of nivolumab in patients with advanced hepatocellular carcinoma (HCC): CA209-040. Presented at: 51st Annual Meeting of American Society Of Clinical Oncology. Chicago, IL, USA, 29 May-2 June 2015.

-. Early results from this Phase I/II study of nivolumab in patients with HCC, many of who were previously treated with sorafenib, are promising. Of note, a Phase III trial is now being initiated, and this study protocol has since been modified to include a nivolumab/ipilimumab combination arm.

87 Larkin J, Chiarion-Sileni V, Gonzalez R et al. Combined nivolumab and ipilimumab or monotherapy in untreated melanoma. N. Engl. J. Med. 373(1), 23-34 (2015).

88 Zhao W, Gu YH, Song R, Qu BQ, Xu Q. Sorafenib inhibits activation of human peripheral blood T cells by targeting LCK phosphorylation. Leukemia 22(6), 1226-1233 (2008).

89 Motoshima T, Komohara Y, Horlad H et al. Sorafenib enhances the antitumor effects of anti-CTLA- 4 antibody in a murine cancer model by inhibiting myeloid-derived suppressor cells. Oncol. Rep. 33(6), 2947-2953 (2015).

90 Chen Y, Ramjiawan RR, Reiberger $\mathrm{T}$ et al. CXCR4 inhibition in tumor microenvironment facilitates anti-programmed death receptor-1 immunotherapy in 
sorafenib-treated hepatocellular carcinoma in mice. Hepatology 61(5), 1591-1602 (2015).

-• Preclinical study providing mechanistic evidence of a hypoxia-mediated immunosuppressive effect by sorafenib in the tumor microenvironment, which was reversible via immune modulatory agents. This provides a rational for combination of sorafenib and checkpoint inhibitors.

91 Morales-Kastresana A, Sanmamed MF,

Rodriguez I et al. Combined immunostimulatory monoclonal antibodies extend survival in an aggressive transgenic hepatocellular carcinoma mouse model. Clin. Cancer Res. 19(22), 6151-6162 (2013).

92 Gauttier V, Judor JP, Le Guen V, Cany J, Ferry N, Conchon S. Agonistic anti-CD137 antibody treatment leads to antitumor response in mice with liver cancer. Int. J. Cancer 135(12), 2857-2867 (2014). 